\title{
LARYNGEAL STENOSIS WITH TRACHEOSTOMY TREATED BY HEMI-LARYNGECTOMY AND RECONSTRUCTION OF THE LARYNGEAL ORIFICE
}

\author{
BY \\ H. S. SHARP \\ From The Hospital for Sick Children, Great Ormond Street, London
}

(RECEIVED FOR PUBLICATION NOVEMBER 30, 1955)

The patient, a girl, $2 \frac{1}{2}$ years old, was born in Jamaica, the birth weight being $7 \frac{1}{2} \mathrm{lb}$. Pregnancy was normal, with the delivery at full term. There was no dyspnoea or cyanosis at birth. Stridor and attacks of "cyanosis" started soon after birth, especially with feeds, culminating with a very severe attack at 11 months. A direct laryngoscopy was performed, and the larynx was 'probed'. The condition deteriorated, and an immediate tracheostomy was performed.

The child arrived in England at the end of June, 1955, when she was admitted to the Victoria Hospital for Children, Tite Street, London. Here a direct laryngoscopy was performed by Mr. Pereira, when a very stenosed larynx was seen, with no details of the true cords observed.

On July 19, 1955, she was transferred to The Hospital for Sick Children, Great Ormond Street. On admission, she appeared in good general health. The tracheostomy tube was in situ.

On July 21, 1955, a direct laryngoscopy was performed, and the earlier findings were confirmed. The laryngeal orifice was extremely small, and no details of the left true cord could be seen at all. The whole of this side of the larynx appeared to be fibrosed. By angling the laryngoscope, the right true cord was seen in part, and this appeared to be normal. The epiglottis was normal. It was quite impossible to pass even a suckling bronchoscope through the larynx-a probe was the only instrument it would admit.

A trial of respiration without the tracheostomy tube was then made, but it was obvious that the child could not breathe without the tracheal stoma being patent, and the tube was reinserted.

It appeared that the condition of the larynx was initially one of congenital stridor, and that this had been made worse by the probing at the first laryngoscopy. This probably produced submucosal damage to the left side of the larynx, with consequent fibrosis and organic narrowing. Certainly the laryngoscopic appearance was new to me, and did not conform with the various types of abnormality which cause the syndrome of congenital stridor. The Wassermann reaction was negative.

The problem this case presented was of considerable magnitude. If nothing were done, the child would develop without speech, and with a permanent tracheostomy throughout her years of development. On the other hand, any reconstructive operation on the larynx would be much easier at a later date when the field of operation would be so much larger.

If a reconstructive operation were attempted at once, it was realized that it would be a somewhat difficult procedure, but if success were achieved, it would be obviously of great benefit to the child. The hoped-for outcome was for a functioning larynx, with a usable though weak voice and a normal airway, together with closure of the tracheostomy. It was thought that probably the larynx could be made large enough for normal respiration, but whether or not the voice would be restored was unpredictable.

These various points were discussed with the mother, and she agreed that an immediate reconstructive operation should be undertaken. Depending on its outcome, it was intended to close the tracheostomy at a later date. Accordingly, on July 29, 1955, the procedure was embarked upon. Under general anaesthesia a vertical incision was used through the skin and superficial tissues, eventually opening the larynx in the midline. The cricoid cartilage was cut through, and the trachea as far down as the tracheostomy opening. The right true cord was identified, and appeared normal. The left side of the larynx showed no proper structure, and was adherent across the anterior commissure but not in a web formation. The whole of the left side of the larynx was separated in the submucosal layer from the thyroid cartilage and removed, leaving only the cartilaginous surface as the left laryngeal boundary. The right side of the larynx was not interfered with. A No. 1 triangular portex tube (Negus pattern), cut to 2 in. in length, was then inserted in the wound from the cephalad point of the thyroid cartilage to the tracheostomy tube in the trachea; the tube was used with the base placed anteriorly. This was covered on the anterior and lateral aspects with a skin graft of appropriate size, the appearance then being as in the figure. No attempt was made to close the thyroid and cricoid cartilage over this or the trachea. The strap muscles of the neck were closed over the skin graft, and the superficial tissues and skin over these. A silver wire was inserted through the thyroid 
cartilage and portex tube to retain the latter in position. An oesophageal tube was placed through the nose for feeding purposes, the tracheostomy tube being still in place. The child stood the procedure well, and was returned to the ward in good condition. She was

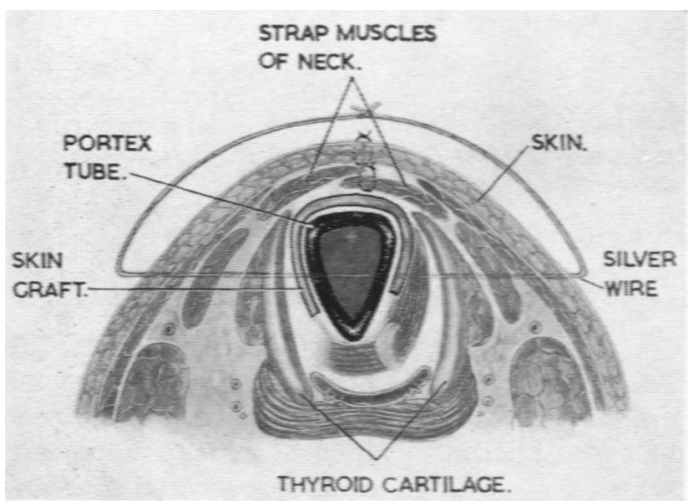

nursed for 24 hours in an oxygen tent under constant supervision, with suction as necessary. There was no pyrexia, and feeding presented no difficulty. The child was under full antibiotic therapy.

On August 5, 1955, the portex tube was removed by direct laryngoscopy, the silver wire stay suture having been first withdrawn. The tube came away easily with no adherent skin. The laryngeal aperture appeared adequate, but the tracheostomy tube was not removed.

On August 10,1955, it was seen that the child was breathing through the nose, so the tracheostomy tube was removed. By August 20,1955, the tracheal stoma had almost closed. The child made a 'noise' when crying, so that it could be assumed that there was going to be some form of voice. However, on August 30, she contracted an upper respiratory infection. There was a return of laryngeal stridor, and the tracheostomy tube was reintroduced.
On September 8 a direct laryngoscopy was performed. The larynx looked normal on the right side, and posteriorly the inlet was satisfactory. There were a few granulations on the mucosa anteriorly on the left side and these were removed and the laryngeal aperture gently stretched by opening the jaws of a pair of Patterson's forceps in it. On September 20 the tracheostomy tube was removed, and there has been no further trouble since this date.

On October 6 a further direct laryngoscopy and tracheoscopy was performed. The laryngeal aperture was satisfactory and the tracheal lumen adequate and the right true cord was seen to move. The anterior wall of the trachea appeared very injected (this was the area composed of skin graft), but there were no granulations. The tracheostomy orifice had closed spontaneously.

This patient has now returned home and it is hoped that she will shortly catch up in speech with children of her age.

It seems that the risks undertaken in operating on this child at this age were justified by the outcome. The operating field is extremely small, but provided adequate care is taken, the procedure is not particularly difficult. With this patient it may be necessary at a later date to free the skin of the neck from the underlying fibrous tissue, since the original tracheostomy scar was very adherent.

It was interesting to find that the tracheostomy stoma closed spontaneously; this is most unusual in a child so young, and in whom the tracheostomy tube had been in position for one and a half years. Provided that the laryngeal aperture can be made adequate, it seems that this favourable outcome can be expected.

I should like to express my thanks to Mr. Pereira for referring this case to me, to Dr. Sheila Anderson for her skilful anaesthesia on each occasion and to my Registrar, Mr. Halfhide, for his help and encouragement. The care of the sister and nurses in the ward contributed in no small measure to the successful outcome. 